Meta

Journal des traducteurs

Translators' Journal

\title{
L'humour est-il traduisible? Des notes sur un extrait de Babbitt
}

\section{Jacques Laroche}

Volume 34, numéro 1, mars 1989

Humour et traduction

Humour and Translation

URI : https://id.erudit.org/iderudit/003402ar

DOI : https://doi.org/10.7202/003402ar

Aller au sommaire du numéro

Éditeur(s)

Les Presses de l'Université de Montréal

ISSN

0026-0452 (imprimé)

1492-1421 (numérique)

Découvrir la revue

Citer cet article

Laroche, J. (1989). L'humour est-il traduisible? Des notes sur un extrait de Babbitt. Meta, 34(1), 15-19. https://doi.org/10.7202/003402ar d'utilisation que vous pouvez consulter en ligne.

https://apropos.erudit.org/fr/usagers/politique-dutilisation/ 


\section{L'HUMOUR EST-IL TRADUISIBLE ? DES NOTES SUR UN EXTRAIT DE BABBITT}

JACQUES LAROCHE

New Mexico State University, Las Cruces (U.S.A.)

Depuis une quinzaine d'années, $j$ 'enseigne la traduction littéraire dans les universités américaines. C'est un sujet qui semble tout naturel à un Français dont la formation linguistique a consisté essentiellement à traduire des textes français, grecs et latins. L'idée n'a pas le même caractère d'évidence pour mes collègues américains formés à l'école béhavioriste. Ces pragmatistes se demandent quelle utilité pratique ça peut bien avoir. La traduction technique, encore..., disent ces professeurs de littérature. Sans me soucier outre mesure de ces apôtres de la rentabilité, je continue à entraîner mes étudiants à la traduction, version et thème, en employant la terminologie de l'excellente Stylistique comparée du français et de l' anglais (J.-P. Vinay \& J. Darbelnet, Paris: Didier, 1958). Après chaque devoir, mes étudiants reçoivent un corrigé raisonné justifiant les choix faits parmi les traductions possibles.

Un texte humoristique est-il utilisable dans cette discipline? Dans n'importe quelle traduction littéraire, le traducteur peut se donner à coeur joie la satisfaction de décortiquer le style de l'auteur (les critiques littéraires à la page parlent de «déconstruction»). C'est une bonne occasion de montrer que nul genre n'est spécifique d'une culture et que tout peut se traduire, y compris l'humour. L'Anglo-Saxon n'a pas plus naturellement le sens de l'humour que le Français ne manque de tête épique...

L'exemple donné ici est un extrait de Babbitt (New York: Harcourt, Brace à World, 1922, pp. 23-24). On se rappelle cette satire brûlante des notables du Middlewest où Sinclair Lewis peint l'esprit «Rotary Club». Les notes qui suivent représentent le corrigé distribué à mes étudiants après ce thème difficile (les traducteurs savent bien que «thème difficile» est une tautologie). Il va sans dire que le texte en a été sensiblement amendé pour des lecteurs qui ne partagent pas la merveilleuse innocence linguistique de mes étudiants. D'une part, les notes que je distribue sont en anglais puisqu'une analyse en français serait pour des anglophones une pétition de principe. Je sais, d'autre part, que cette satire sociale n'a pas la même originalité pour des érudits internationaux que pour mes âmes pures du Southwest, mais j'ai souhaité garder le réalisme de l'exercice.

Voici le texte, où les locutions en caractères gras renvoient à l'analyse verbale:

\section{AN ALL-AROUND EXPERT}

On the other side of Babbitt lived Howard Littlefield, Ph.D., in a strictly modern house whereof the lower part was dark red tapestry brick, with a leaded oriel, the upper part of pale stucco like spattered clay, and the roof red-tiled. Littlefield was the Great Scholar of the neighbourhood; the authority on everything in the world except babies, cooking, and motors. He was a Bachelor of Arts of Blodgett College, and a Doctor of Philosophy in Economics of Yale. He was the employment-manager and publicity counsel of the Zenith Street Traction Company. He could, on a ten hours' notice, appear before the Board of Aldermen or the State Legislature and prove, absolutely, with figures all in rows and with precedents from Poland and New Zealand, that the street-car company loved the Public and 
yearned over its employees; that all its stock was owned by Widows and Orphans; and that whatever it desired to do would profit property owners by increasing rent values, and help the poor by lowering rents. All his acquaintances turned to Littlefield when they desired to know the date of the battle of Saragossa, the definition of the word «sabotage», the future of the German Mark, the translation of "hinc illae lachrimae», or the number of products of coal tar. He awed Babbitt by confessing that he often sat up till midnight reading the figures and footnotes in Government reports, or skimming (with amusement at the author's mistakes) the latest volumes of chemistry, archeology and ichthyology.

But Littlefield's great value was as a spiritual example. Despite his strange learnings, he was as strict a Presbyterian and as firm a Republican as George Babbitt. He confirmed the businessmen in their faith. Where they knew only by passionate instinct that their system of industry and manners was perfect, Dr. Howard Littlefield proved it to them, out of history, economics, and the confessions of reformed radicals.

Lewis, Sinclair (1922): Babbitt.

\section{REMARQUES GÉNÉRALES}

"The All-Around Expert» a été choisi parce que l'original ne comporte pas de difficultés de traduction très graves, mais, au contraire, un nombre de problèmes limités qu'il faudra régler séparément.

Il y a beaucoup de «couleur locale» : diplômes, institutions et comportements qui seront étrangers à bien des lecteurs francophones. Si la traduction était spécifiquement destinée au marché canadien, on pourrait attendre une plus grande connaissance de la culture américaine, mais on est censé s'adresser au lecteur francophone en général.

Grâce à son humour bon-enfant, le texte représente une vision amusée de la culture de la vie bourgeoise américaine qui ne se démode pas : dans les années 70 on y voyait une image désabusée du consultant universitaire du secteur privé et aujourd'hui, on jurerait lire le portrait d'un conseiller présidentiel! Le stéréotype du grand savant de province n'est pas inconnu de la littérature française, c'est le cas de M. Homais dans Madame Bovary.

Le texte ne fait aucune critique directe de Littlefield. Lewis nous invite malicieusement à voir un hypocrite de l'œil naïvement admiratif de Babbitt et à en conclure que les gens vulgaires méritent amplement les héros qu'ils se créent !

La description technique du domicile de Littlefield nous rappelle que Babbitt est «dans» l'immobilier et considère un bâtiment techniquement pour autant que l'obscurité du jargon contribue à la valeur de la propriété.

Il faudra prendre garde à mesurer soigneusement le niveau d'ironie de la traduction afin d'éviter la sursaturation et de verser, nous aussi, dans le pharisaïsme moralisateur qui est précisément la cible de Lewis!

\section{ANALYSE VERBALE}

\section{«An All-Around Expert»}

L'antithèse appuyée du titre (en anglais, oxymoron) est rendue en français par «Un spécialiste universel».

\section{Ph D}

Les lecteurs français ignoreront souvent ce que ce diplôme représente sur le plan social ou académique. «Docteur de l'Université» est à peu près équivalent et indique plus clairement que le docteur Littlefield que l'intéressé n'est pas médecin. 


\section{Tapestry brick}

Probablement équivalent de brick veneer dans le jargon des maçons des années 20 . Ce qui compte est de donner une impression de bagout technique obscur. «Brique de parement» m'est également obscur...

\section{Leaded oriel}

«Fenêtre-vitrail en encorbellement». Babbitt est en train de décrire ici l'équivalent du pavillon de la banlieue parisienne à la même époque.

\section{Everything in the world}

Modulation avec une métaphore équivalente : «tout ce qui existe sous le soleil».

\section{Babies, cooking, motors}

Comme en anglais britannique, motor représente plus souvent «voiture» que «moteur» en 1920 , c'est une synecdoque (partie pour le tout). Transposition concret/abstrait dans babies = «puériculture». Remarquons que Littlefield se désolidarise prudemment des activités trop peu masculines ou trop «manuelles»...

\section{BA, Doctor in Philosophy of Economics, Blodgett College}

«Licence ès lettres», «Doctorat en science économique». Le lecteur français serait déconcerté par cette association d'économie et de philosophie. Je préfère garder Blodgett College, car j'ignore si Blodgett est un éponyme ou un toponyme.

\section{Publicity Counsel}

En France aussi, c'est aujourd'hui «public relations», mais ce n'était pas encore le cas dans les années 20.

\section{Street Traction Company}

Les transports publics sont l'affaire des «Compagnies de transport urbain». Un street car était en France «un tramway», cas d'emprunt erroné puisque ce mot signifie «télécabine» pour les Américains.

\section{State Legislature}

En France, il n'y a pas d'équivalent à «l'Assemblée d'État». Dans d'autres pays francophones fédéraux il y en a, par exemple le «Synode cantonal» en Suisse, mais, même pour des lecteurs helvétiques, ce choix perdrait la couleur locale.

\section{... that the street-car company ..., rents}

La satire du positive thinking est ici audacieuse et pourrait aisément sembler forcée. Les absurdités doivent être visibles sans insulter l'intelligence du lecteur. Naturellement, la compagnie ne peut montrer son amour pour ses employés qu'en haussant ses tarifs et on a peine à voir comment l'augmentation des valeurs locatives profiterait aux locataires ! On devra laisser les contradictions telles quelles et souhaiter que le pot-pourri de style bureaucratique et de mots sentimentaux (love, yearn for, Widows and Orphans) ne rende pas le texte trop naivement revendicatif. 


\section{Sabotage}

Les lecteurs français n'admettront jamais qu'un simple mot français soit jugé exotique. On ne peut guère non plus employer un mot anglais. Il faudra donc recourir à une adaptation et prendre un terme d'une troisième langue notoire à l'époque de référence. «Bolchévique» (les anglophones noteront l'orthographe de la translitération française) donnait de délicieux frissons aux Français aussi bien qu'aux Américains dans les années 20 !

\section{Strange Learnings}

Il nous faut rendre l'idée qu'érudition si ésotérique (le pluriel learnings est révélateur) dépasse le cadre conceptuel du Rotary Club de Zénith et que les doctrinaires bibliques de la province américaine pourraient y renifler comme un effluve de soufre ! «Qui sentait le fagot» (comme les hérétiques condamnés au bûcher) est trop évocateur d'intolérance religieuse; or l'intolérance babbittienne est bien plus universelle. Je suggère «peu catholique» qui signifiait bien hérétique à l'origine, mais aujourd'hui s'applique à ce qui est tout simplement hétérodoxe. Que faire du conflit «peu catholique» / «Presbytérien»? Justement, si John Knox se flattait d'être «peu catholique», Babbitt, qui ne se pique pas de casuistique, y verrait purement une rupture de la routine sacro-sainte, et c'est probablement là qu'est sa vraie religion... Littlefield est un érudit, mais ses amis lui passeront cette excentricité tout de même un peu déplacée.

\section{Reformed Radicals}

Dans la sémantique française des années 20 , «radical» avait perdu son sens de «révolutionnaire», associé qu'il était au «Parti radical-socialiste», un parti super petit-bourgeois et ultracentriste (encore des oxymorons). Pour un Américain, d'autre part, reformed a un aspect mélioratif qui n'est pas évident pour les enfants de «la fille aînée de l'Église»! Nous devons donc utiliser un mot décrivant un changement de mal en bien. «Converti» ne préjuge pas que l'affiliation précédente était coupable. «Repentant» implique que le changement est en cours. Pour faire des conférences et recueillir la faveur des conservateurs, il faut avoir passé ce stade et être devenu un «repenti» sûr et digne de confiance...

Voici maintenant la traduction que je propose:

\section{UN SPÉCIALISTE UNIVERSEL \\ Traduit par Jacques Laroche}

L'autre voisin de Babbitt était Howard Littlefield, Docteur de l'Université, qui avait une maison résolument moderne, avec un soubassement de brique de parement rouge foncé, une fenêtre-vitrail en encorbellement, un haut de stuc clair imitation crépi et un tôt de tuiles rouges.

Littlefield était le grand savant du quartier, une autorité universelle sur tout ce qui existait sous le soleil, à l'exception de la puériculture, la cuisine et la mécanique automobile. Il avait une Licence ès lettres de Blodgett College et un Doctorat en science économique de Yale. C'était le directeur du personnel et le porte-parole de la Compagnie des Transports Urbains de Zénith. Moyennant un préavis de dix heures, il pouvait se présenter devant le Conseil municipal ou l'Assemblée d'État et prouver sans conteste, en alignant tous ses chiffres et en citant des précédents en Pologne et en Nouvelle-Zélande, que la Compagnie des tramways portait au public un amour sans bornes et chérissait ses employés, que toutes ses actions étaient détenues par la veuve et l'orphelin, et que les projets de la Compagnie augmenteraient la valeur locative, au plus grand profit des propriétaires immobiliers, tout en secourant les pauvres en diminuant leur loyer. Toutes les relations de Littlefielf faisaient appel à lui chaque fois qu'on voulait des renseignements sur la date de la bataille de Saragosse, la définition du mot «bolchévique», le sort du Deutsch Mark, la traduction de "hinc illae lachrimae» ou la liste des sous-produits du goudron. Il impressionnait Babbitt quand il avouait 
avoir souvent veillé jusqu'à minuit à lire les chiffres et les notes des statistiques officielles, ou à parcourir (en souriant aux naïvetés de l'auteur) les plus récents ouvrages de chimie, d'archéologie et d'ichtyologie.

Mais c'était comme exemple moral que Littlefield se montrait le plus précieux. Malgré son érudition peu catholique, c'était un Républicain aussi intransigeant et un presbytérien aussi strict que Babbitt. Il confirmait les négociants dans leur Credo. Là où seule leur ferveur instinctive leur disait que leur code commercial et social était sans reproche, le Docteur Littlefield savait le leur prouver, par la vertu d'exemples historiques et économiques et les autocritiques de révolutionnaires repentis.

D'après Sinclair Lewis (1922): Babbitt. 